\title{
The pilot study of object following for automated guided vehicle
}

\author{
Jieh-Shian Young and Cheng-Yan Lin \\ Institute of Vehicle Engineering, National Changhua University of Education, \#1, Jin-De Rd, Changhua 500, Taiwan
}

\begin{abstract}
This paper aims at the pilot study of the object following for automated guided vehicle (AGV). The proposed approach utilizes the laser range finder (LRF) to detect the distances of all obstacles surrounded in a specified angle range. The AGV can autonomously follow the assigned object according to the rational reflection intensity and range of the object sensed from LRF. The cruise speed control for a moving object depending on the distance between AGV and this object is considered since it is one of the crucial parameters for object following. This paper also discusses the influences of the sensor delay which is an indispensable issue in both control law and control logic. This pilot study employs a down scaled AGV to verify the feasibilities of the steering, accelerating, braking, and object following algorithms developed for AGVs.
\end{abstract}

\section{Introduction}

In 2009, the US National Highway Safety Administration began studying collision warning system specification. In 2011, the European Commission adopted a resolution and began to promote pre-brake system. In 2013, all commercial vehicles within Europe must be installed the pre-crash brake system. In 2015, all new cars not just commercial vehicles within Europe must be installed forward collision warning (FCW).

Many studies show that when vehicles have forward collision warning or pre-crash brake assist or autonomous pre-crash brake systems, can increase driving safety and reduce the severity of the injury $[1,2]$.

The automobile industry needs not only safe structures but also active safety. Moreover, traffic in Taiwan is highly congested. It may cause the car accidents as drivers do not pay attentions due to the traffic jams, tired driving, etc. In case vehicles with automatic brakes and automatic following system can raise the traffic efficiency and reduce the driving hazards. The realization of active safety systems can be achieved through the information collected by sensors that identify front environment and obstacles. Furthermore, an appropriate control mechanism applied to vehicle safety is indispensable.

Many studies have utilized a variety of techniques based on difference sensors to achieve the object following functions, such as radio-frequency identification (RFID)[3], Kinect sensor (computer vision)[4], laser range finder (LRF) [5, 6], etc. Some of them utilize multiple sensors[7]. This study proposes a technique which adopts only LRF sensor by its range information and reflection intensity. This paper shows the results of the pilot study for its feasibility of object following function in a vehicle verified by an automated guided vehicle (AGV).

\section{Preliminaries for experiment setup}

\subsection{The AGV}

The AGV adopted in this study is one of the products from Play Robots Inc., or 4W4D Combo AGV as shown in Figure 1. The AGV has size of $42 \times 32 \times 17 \mathrm{~cm}$. The wheels of the AGV are equipped by four $12 \mathrm{~V}$ DC motors. Its maximal moving speed is approximate to $1.1 \mathrm{~m} / \mathrm{s}$. The maximal payload of the AGV is $5 \mathrm{~kg}$. This AGV is with the maximal climbing angle of $40^{\circ}$. The data communication medium for the AGV is the Bluetooth by which the control commands can be received and some requests can be also transmitted. Table 1 lists the brief specifications of the AGV. The laptop personal computer (PC) can play as a main control role for the AGV since the maximal payload weight is much less than $5 \mathrm{~kg}$. In addition, $\mathrm{PC}$ can receive the distance information from LRF through Ethernet by proper software programming.

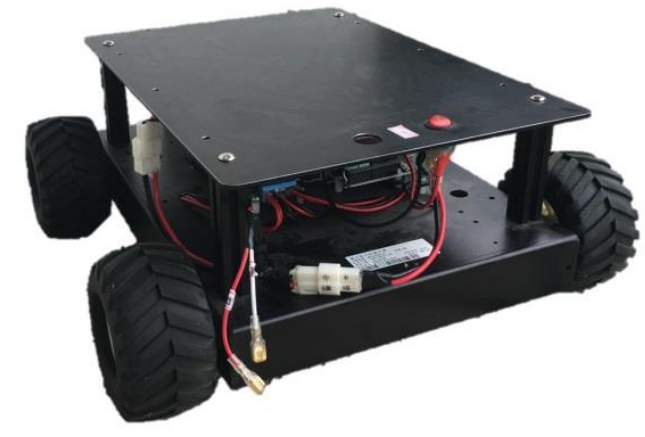

Figure 1. The 4W4D Combo AGV[8]. 
Table 1. The specifications of the 4W4D Combo AGV[8].

\begin{tabular}{||c|c|}
\hline Model & 4W4D-combo \\
\hline Supply voltage & 6VDC/12VDC battery \\
\hline Dimension & $42 \times 32 \times 17 \mathrm{~cm}$ \\
\hline Power source & DC $12 \mathrm{~V}$ motor*4 \\
\hline Max. moving speed & $1.1 \mathrm{~m} / \mathrm{sec}$ \\
\hline Max. climbing angle & $40^{\circ}$ \\
\hline Max.load & $5 \mathrm{~kg}$ \\
\hline Data medium & Bluetooth \\
\hline
\end{tabular}

The AGV utilizes the Arduino UNO micro control board and can receive commands from controller via Bluetooth module. The motors revolution rate of the AGV can be control by discretized speed command values ranged from 0 to 127 . Figure 2 sketch the relation between speed command values and the speeds of the AGV. $\mathrm{Y}=\mathrm{X}-2.3$ is the linear approximate to the test data for different speed commands where $X$ is the speed command and $\mathrm{Y}$ is the speed of the AGV.

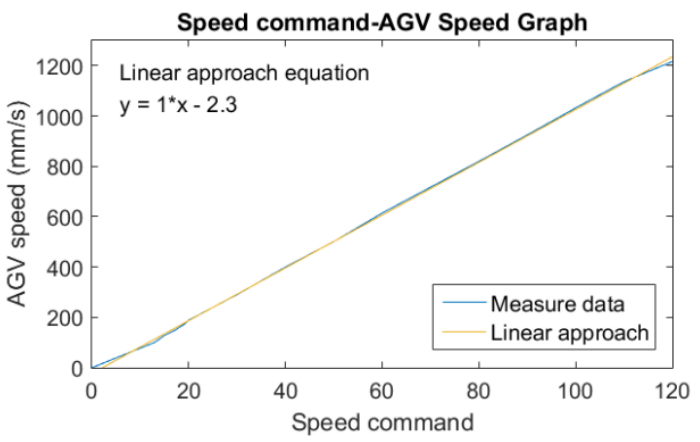

Figure 2. Relation between speed command and AGV speed.

\subsection{The laser range finder (LRF)}

There are many methods to measure the distance in applications such as ultrasonic sensors, computer vision with image process, LRF, etc. Table 2 lists the comparisons for these sensors. The computer vision approach has lower cost. However, their accuracies and computation time are not as good as the LRF. In addition, they are very sensitive to the environment such as image background. For the sake of the fast and accurate requirements, LRF is adopted in this paper.

Table 2. Comparisons for different sensors.

\begin{tabular}{||c|c|c|c|}
\hline & $\begin{array}{c}\text { Computer } \\
\text { Vision }\end{array}$ & $\begin{array}{c}\text { Ultrasonic } \\
\text { Sensor }\end{array}$ & $\begin{array}{c}\text { Laser } \\
\text { Range } \\
\text { Finder }\end{array}$ \\
\hline Error Rate & Low & High & Mid \\
\hline Computing & Fast & Slow & Mid \\
\hline Accuracy & Mid & Low & High \\
\hline
\end{tabular}

The type of the LRF in this study is Hokuyo UST20LX. The distance detected for this kind of LRF is ranged from $0.06 \mathrm{~m}$ to $60 \mathrm{~m}$ with the accuracy of \pm 0.04 $\mathrm{m}$. Its scanning angle is from $-135^{\circ}$ to $135^{\circ}$, or the span angle is $270^{\circ}$ as shown in Figure 3 . The scanning speed of the LRF is $25 \mathrm{~ms} / \mathrm{rev}$ and one step, or the angular resolution, is $0.25^{\circ}$. It is intuitive that the maximal step number is 1080 . The data communication field bus for the LRF is the Ethernet with the specified data communication protocol. In applications, the range of the scanning angle for this LRF can be assigned within $\pm 135^{\circ}$ by command received via Ethernet. Figure 4 shows the LRF of Hokuyo UST-20LX. Table 3 lists its specifications.

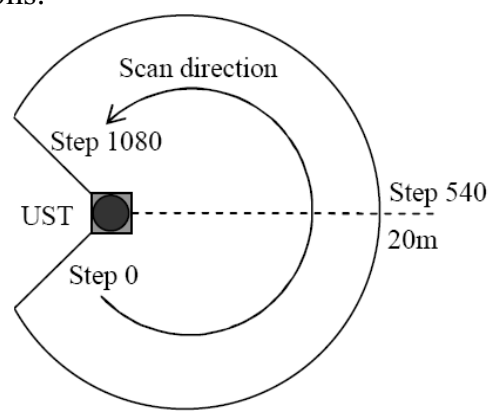

Figure 3. The scanning angle of Hokuyo UST-20LX[9].

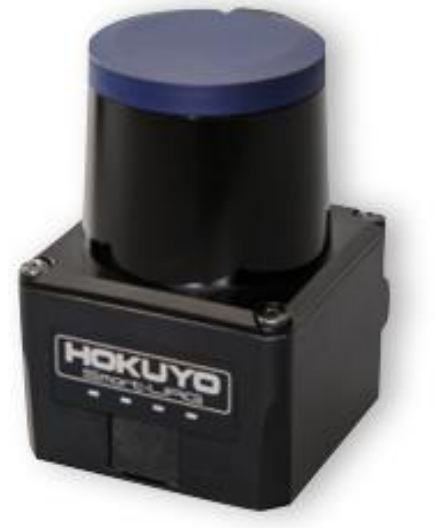

Figure 4. The LRF of Hokuyo UST-20LX[10].

Table 3. The specification of Hokuyo UST-20LX[9].

\begin{tabular}{||c|c||}
\hline Model & UST-20LX \\
\hline Supply voltage & $12 \mathrm{VDC} / 24 \mathrm{VDC}$ \\
\hline Supply current & $<150 \mathrm{~mA}$ \\
\hline Detection range & $0.06 \mathrm{~m}$ to $60 \mathrm{~m}$ \\
\hline Accuracy & $\pm 0.04 \mathrm{~m}$ \\
\hline Scan angle & $270^{\circ}$ \\
\hline Scan speed & $25 \mathrm{~ms}($ at $2400 \mathrm{rpm})$ \\
\hline Angular resolution & $0.25^{\circ}$ \\
\hline Data Medium & Ethernet \\
\hline
\end{tabular}

The Hokuyo UST-20LXLRF data communication medium is Ethernet with Sensor Communication Interface Protocol (SCIP)[11]. Its sensor structure sketched in Figure 5 composes of host part, communication interface part, and target (sensor) part. The controller has to establish a TCP/IP communication mechanism in order to receive the information of the LRF including the distances, reflection intensities, and the corresponding angles. For example, the factory default valuesfor the network address settings are as follows. IP 
address: 192.168.0.10; Subnet Mask: 255.255.255.0; Default Gateway: 192.168.0.1; Port Number: 10940. The typical responses of Hokuyo UST-20LX LRF are illustrated in Figure 6 as the PC transmits the "GE" command which is so-called the distance and intensity acquisition command for Hokuyo UST-20LX. The data in Figure 6 are actually the printable ASCII codes. Each distance datum comprises 3 characters and each character for distance information contains 6 bits. For instance, " $1 \mathrm{Dh}$ " is decoded as $5432 \mathrm{~mm}$. Figures 7 and 8 illustrate the decoding algorithm and the verification software program, respectively.

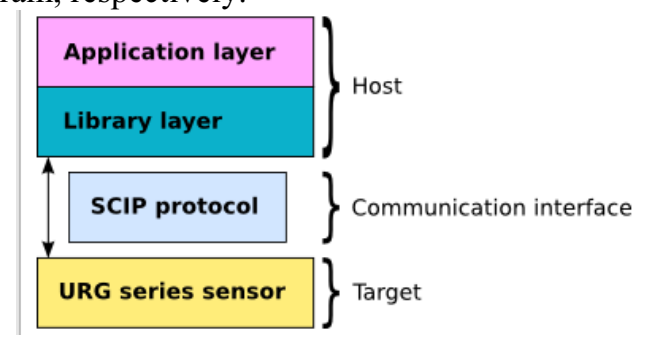

Figure 5. The sensor structure of Hokuyo UST-20LX[11].

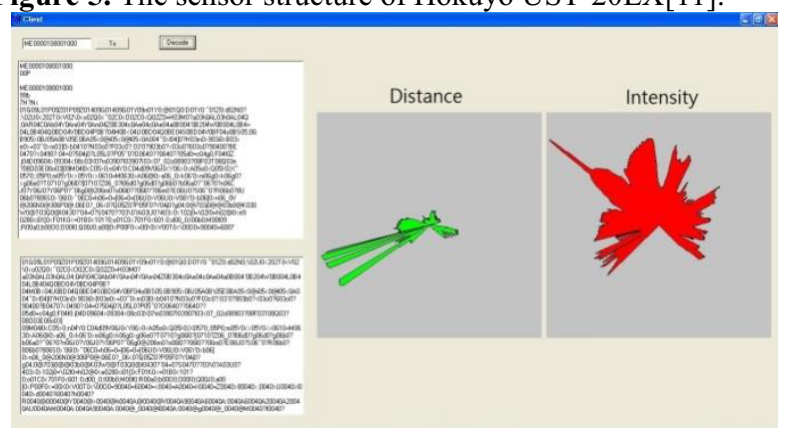

Figure 6. The typical responses of Hokuyo UST-20LX.

$$
\begin{gathered}
\frac{\text { ' } 1 \text { ' }(31 \mathrm{~h}) \text { 'D' }(44 \mathrm{~h}) ' \mathrm{~h} \text { ' }(68 \mathrm{~h})}{\downarrow \text { Subtract } 30 \mathrm{~h}} \\
\frac{1 \mathrm{~h} \quad 14 \mathrm{~h} \quad 38 \mathrm{~h}}{\downarrow \text { Merge }} \\
\frac{000001 \quad 010100 \quad 111000}{\downarrow \text { Decimal Value }} \\
2^{3}+2^{4}+2^{5}+2^{8}+2^{10}+2^{12}=5432 \mathrm{~mm}
\end{gathered}
$$

Figure 7. The distance decoding algorithm[12].

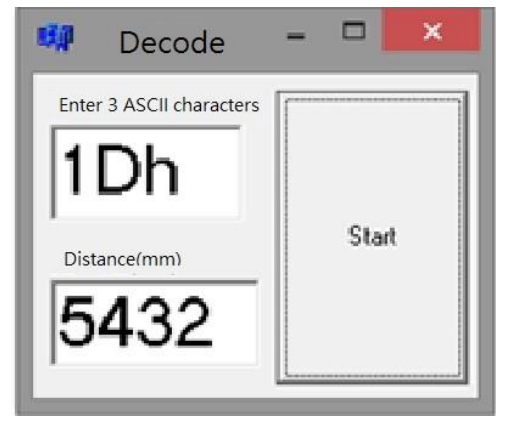

Figure 8. The distance decoding algorithm and the verification software program.

\subsection{AGV control platform}

In this study, the laptop PC served as a steering controller is fixed on the platform as shown in Figure 9. The LRF is installed on the forefront of the AGV. This PC can transmit the control commands to the LRF and can receive the measuring data from LRF viathe Ethernet port of LRF. The laptop PC analyzes the received data from LRF, determines the potential object, and forwards an applicable command to AGV via bluetooth after following a control rule including control law and control logic.

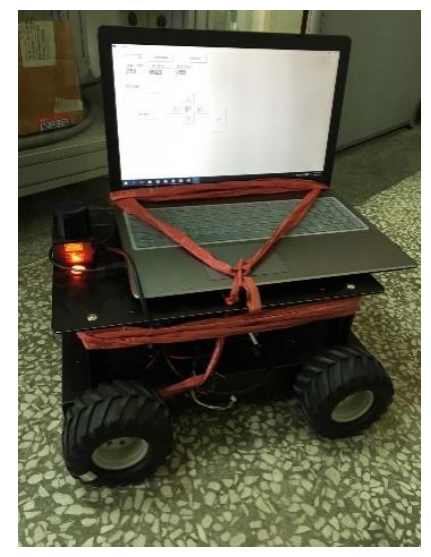

Figure 9. AGV control platform.

LRF provides the distances and intansities reflected from obstacles located within the assigned scanning angles span. The control platform has two main problems. One is that how to recognize the assigned object with the LRF response data. The instansity information is practical if the strongest reflection instansity is detected from the assigned object. The PC, or the steering controller, can then be easy to recognize the potential object by the different reflection intansity patterns or strengths of the object. The AGV can follow the object with the appropriate control strategy based on its corresponding distance and angle. The other problem is that what control strategy can make the object following of AGV more accurate and more efficient. This pilot study aims at the previous studied not only on the communication techniques between PC and LRF and between PC and AGV but the techniques of the LRF information analysis, the potential object determination, applicable control commands generation.

\section{Control specifications}

A properly distance between the object and the AGV has to be kept for the sake of the object following purpose. The proper distance definitely depends on the speed of the object. Figure 10 sketches the proposed relation between distance to target and $\mathrm{AGV}$ speed. Under consideration of avoiding wheel slip, moderate acceleration for the AGV is necessary. In this paper, the final speed will be achieved by separating 10 portionswithin 0.1 second from initial speed. For instance, the initial speed and final speed of AGV are $0.5 \mathrm{~m} / \mathrm{s}$ and $1 \mathrm{~m} / \mathrm{s}$, respectively. The speed commands are sequentially transmitted for every $0.01 \mathrm{~s}$ as $V_{c}=0.4+\frac{(1.0-0.4) n}{10}$ where $V_{c}$ is the speed command and $n$ is ranged from 1 to 10 . 


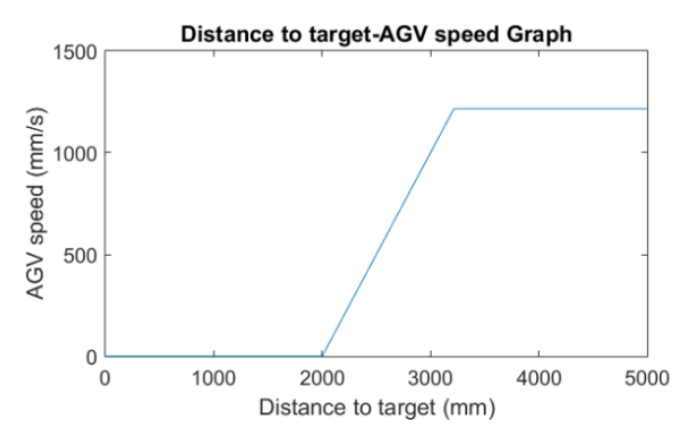

Figure 10. Distance-speed specification for AGV.

The turning mechanism of the AGV is also important for the object following purpose. This paper proposes a similar triangles rule to facilitate the turning of the AGV. Figure 11 schemes the relations of the proposed rule.This rule can be formulated as an equation with turning radius and the speed of the $\mathrm{AGV}$ as follows.

$$
R=\frac{B}{2}\left(\frac{V_{o}+V_{i}}{V_{o}-V}\right),
$$

where $V_{o}$ is the velocity of outward wheels, $V_{i}$ is the velocity of inward wheels, $V$ is the velocity of AGV, $R$ is the turn radius of $\mathrm{AGV}$, and $B$ is width of $\mathrm{AGV}$.

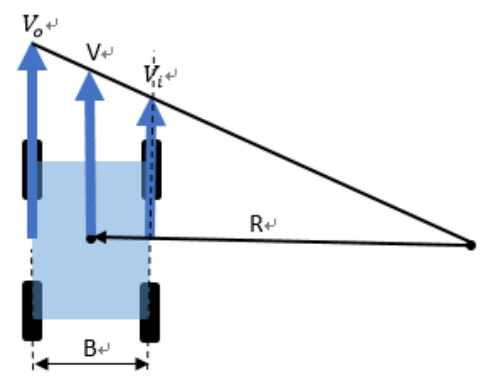

Figure 11. Similar triangle rule for AGV turning.

An accurate path to the target for the AGV is determined by the desired steering radius of the AGV according tothe distance and angle data received from the LRF. Figure 12 sketches the relation between the AGV and the target object where $L$ is the distance between the $\mathrm{AGV}$ and the target object, $R$ is the desired turning radius of $\mathrm{AGV}$, and $\frac{\pi}{2}-\theta$ is the heading angle from the $\mathrm{AGV}$ to target object. In Figure 12, it yields

$$
\frac{R}{\sin \theta}=\frac{L}{\sin (180-2 \theta)} \text {. }
$$

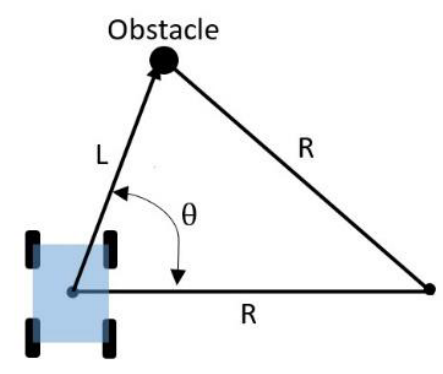

Figure 12. Relation between the AGV and the target object.

\section{Verifications of pilot study}

The steering controller can utilize the laser intensity and distance information from LRF to determine the potential target object. However, the intensity varies from the distance information. It is indispensable to attenuate the distance effect and that will facilitate the determining the target object. Figure 13 demonstrates the target object determining program.

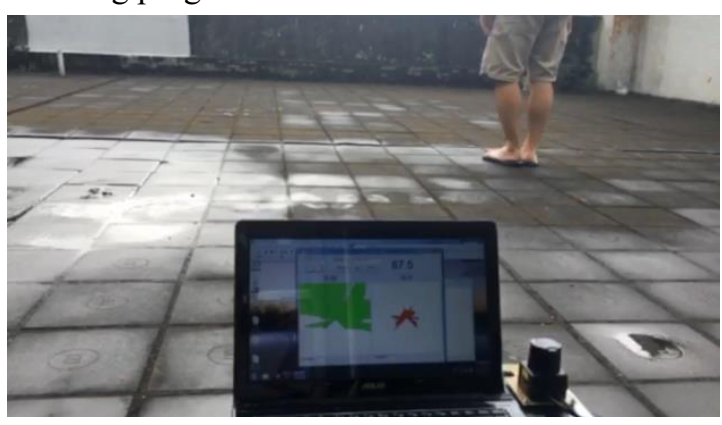

Figure 13. The target object determining program.

The steering control strategy for a straight moving object is one of the most significant verifications for the object following of AGV. The target object will accelerate and decelerate as it moves straight in the verification scenarios. The AGV following the specified target as the target moves straight has been verified but the responses are not quick enough. This paper suggests that the scanning angle of the LRF can be smaller to save the time of decoding since the bottleneck of the computation is the LRF response information decoding.

\section{Conclusion}

This pilot study conveys almost all possible preliminary work for the target object following of AVG. This paper proposes the control specifications such as the relation of the distance and the speed, the similar triangle rule for AGV turning, and the relation between the distance and the turning radius. The future work will be carried out for all possible verifications of the target object followingincluding the target object moves in S-type route in different radii. That will verify the capability of the steering controller for different turning radius.

\section{References}

1. M. Furutani, presented at the Proceedings of Convergence International Congress \& Exposition On Transportation Electronics, US, 2004 (unpublished).

2. K. D. Kusano and H. C. Gabler, IEEE Transactions on Intelligent Transportation Systems 13 (4), 15461555 (2012).

3. M. Kim, N. Y. Chong, H.-S. Ahn and W. Yu, presented at the Proceedings of The 3rd annual IEEE Conference on Automation Science and Engineering (IEEE CASE 2007), Scottsdale, AZ, USA, 2007 (unpublished). 
4. D. Calisi, L. Iocchi and R. Leone., presented at the VISAPP (Workshop on on Robot Vision), Barcelona, Spain, 2007 (unpublished).

5. E.-J. Jung, J. H. Lee, B.-J. Yi and S. i. Yuta, IEEE Transactions on Mechatronics 19 (6), 1963 - 1976 (2014).

6. W. Chung, H. Kim, Y. Yoo, C.-B. Moon and J. Park, IEEE Transactions on Industrial Electronics 59 (8), 3156-3166 (2012).

7. N. Bellotto and $\mathrm{H}$. Hu, IEEE Transactions on Systems, Man, and Cybernetics, Part B: Cybernetics 39 (1), 167-181 (2009).

8. (2016). Play Robot. Available: http://www.playrobot.com/robotics/666--email.html
9. Kamitani, Utsugi, Kamon and Yamamoto. (2014, Scanning laser range finder smart-URG mini UST20LX(UUST004) specification.

10. (2009). Hokuyo. Available: http://www.hokuyoaut.jp/02sensor/07scanner/ust_101x_201x.html

11. H. Fukuda, J. Fujimoto, D. Huang, K. Kimoto, M. Kristou, M. Nagata, K. Matsuo, M. Musashi, T. Momo, S. Watanabe and F. Wada. (2016). URG Network. Available: https://sourceforge.net/p/urgnetwork/wiki/Home/

12. Aoki and Hosoda. (2014, Communication protocol specification for UST series 10LX/20LX. 